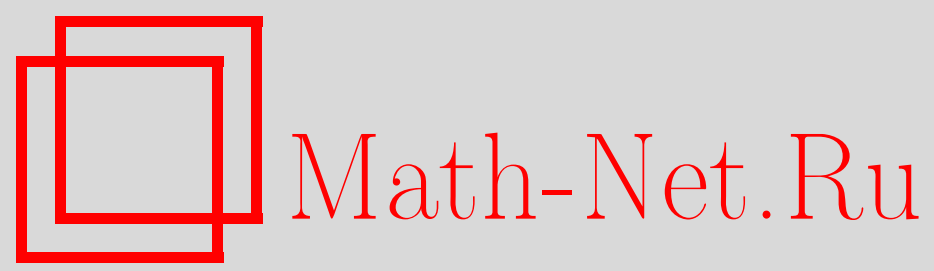

Ю. В. Шеретов, Об общих точных решениях стационарной системы НавьеСтокса и квазигидродинамической системы, не удовлетворяющих уравнениям Эйлера, Вестник ТвГУ. Серия: Прикладная математика, 2017, выпуск 2, 5-15

DOI: https://doi.org/10.26456/vtpmk169

Использование Общероссийского математического портала Math-Net.Ru подразумевает, что вы прочитали и согласны с пользовательским соглашением

http: //www. mathnet.ru/rus/agreement

Параметры загрузки:

IP : 54.224 .135 .184

26 апреля 2023 г., 10:32:47 


\section{МАТЕМАТИЧЕСКОЕ МОДЕЛИРОВАНИЕ, ЧИСЛЕННЫЕ МЕТОДЫ И КОМПЛЕКСЫ ПРОГРАММ}

УДК $517.95,532.5$

\section{ОБ ОБЩИХ ТОЧНЫХ РЕШЕНИЯХ СТАЦИОНАРНОЙ СИСТЕМЫ НАВЬЕ-СТОКСА И КВАЗИГИДРОДИНАМИЧЕСКОЙ СИСТЕМЫ, НЕ УДОВЛЕТВОРЯЮЩИХ УРАВНЕНИЯМ ЭЙЛЕРА}

Шеретов Ю.В.

Тверской государственный университет, г. Тверь

Поступила в редакиию 21.03.2017, после переработки 27.04.2017.

Построены три семейства точных решений, общих для стационарной системы Навье-Стокса и соответствующей квазигидродинамической системы. Эти решения не удовлетворяют уравнениям Эйлера. Приведены конкретные примеры решений, описывающих течения вязкой жидкости. Дана их физическая интерпретация.

Ключевые слова: системы Навье-Стокса и Эйлера, квазигидродинамические уравнения, точные решения.

Вестник ТвГУ. Серия: Прикладная математика. 2017. № 2. С. 5-15.

\section{Введение}

Первые точные решения системы Навье-Стокса для вязкой несжимаемой жидкости, не удовлетворяющие уравнениям Эйлера, были построены в середине XIX века Джорджем Габриэлем Стоксом. Самые известные из них описывают течения Пуазейля в плоском канале и трубе [1], [2]. В XX и XXI веках это направление интенсивно развивалось. Обзоры полученных результатов представлены в научной литературе.

В [3] была предложена еще одна система уравнений, получившая название квазигидродинамической (КГД). Эта система является диссипативной и имеет глубокие связи с классическими моделями Эйлера и Навье-Стокса. Результаты теоретических исследований КГД системы для слабосжимаемой вязкой жидкости представлены в монографиях [4], [5], а также в статье [6]. В частности, подробно изложены методы нахождения ее точных решений. На основе КГД системы конструировались численные методы расчета течений вязкой жидкости как в двумерном [7], так и в трехмерном случае [8].

В настоящей работе построены три семейства точных решений, общих для стационарных систем Навье-Стокса и КГД, но не удовлетворяющих уравнениям Эйлера. Приведены конкретные примеры решений, описывающих течения вязкой жидкости, и дана их физическая интерпретация. 


\section{1. Стационарная система Навье-Стокса для вязкой несжимаемой жид- кости и ее точные решения}

Классическая система Навье-Стокса в динамике вязкой несжимаемой жидкости для стационарных течений имеет вид

$\operatorname{div} \vec{u}=0$,

$$
\operatorname{div}(\vec{u} \otimes \vec{u})+\frac{1}{\rho} \nabla p=\nu \Delta \vec{u} .
$$

Влияние внешних массовых сил не учитывается. В записи системы (1.1) - (1.2), замкнутой относительно неизвестных функций - скорости $\vec{u}=\vec{u}(\vec{x})$ и давления $p=p(\vec{x})$, использованы стандартные обозначения из тензорного анализа. Например, диада $(\vec{u} \otimes \vec{u})$ представляет собой тензор-инвариант второго ранга, полученный как прямое тензорное произведение двух одинаковых векторов $\vec{u}$ и $\vec{u}$. Вектор $\vec{x}=\left(x_{1}, x_{2}, x_{3}\right)=(x, y, z)$ задает точку в пространстве $\mathbb{R}^{3}$. Символы $\operatorname{div}$ и $\nabla$ определяют операции дивергенции и градиента, $\Delta \vec{u}$ - лапласиан векторного поля $\vec{u}$. Система содержит две положительные константы - плотность $\rho$ и коэффициент кинематической вязкости $\nu$.

Выпишем уравнения Навье-Стокса (1.1) - (1.2) в декартовых координатах:

$$
\begin{gathered}
\frac{\partial u_{x}}{\partial x}+\frac{\partial u_{y}}{\partial y}+\frac{\partial u_{z}}{\partial z}=0 \\
\frac{\partial\left(u_{x}^{2}\right)}{\partial x}+\frac{\partial\left(u_{y} u_{x}\right)}{\partial y}+\frac{\partial\left(u_{z} u_{x}\right)}{\partial z}+\frac{1}{\rho} \frac{\partial p}{\partial x}=\nu\left(\frac{\partial^{2} u_{x}}{\partial x^{2}}+\frac{\partial^{2} u_{x}}{\partial y^{2}}+\frac{\partial^{2} u_{x}}{\partial z^{2}}\right), \\
\frac{\partial\left(u_{x} u_{y}\right)}{\partial x}+\frac{\partial\left(u_{y}^{2}\right)}{\partial y}+\frac{\partial\left(u_{z} u_{y}\right)}{\partial z}+\frac{1}{\rho} \frac{\partial p}{\partial y}=\nu\left(\frac{\partial^{2} u_{y}}{\partial x^{2}}+\frac{\partial^{2} u_{y}}{\partial y^{2}}+\frac{\partial^{2} u_{y}}{\partial z^{2}}\right), \\
\frac{\partial\left(u_{x} u_{z}\right)}{\partial x}+\frac{\partial\left(u_{y} u_{z}\right)}{\partial y}+\frac{\partial\left(u_{z}^{2}\right)}{\partial z}+\frac{1}{\rho} \frac{\partial p}{\partial z}=\nu\left(\frac{\partial^{2} u_{z}}{\partial x^{2}}+\frac{\partial^{2} u_{z}}{\partial y^{2}}+\frac{\partial^{2} u_{z}}{\partial z^{2}}\right) .
\end{gathered}
$$

Классическая система Эйлера может быть получена из (1.3) - (1.6) предельным переходом при $\nu \rightarrow+0$ :

$$
\begin{gathered}
\frac{\partial u_{x}}{\partial x}+\frac{\partial u_{y}}{\partial y}+\frac{\partial u_{z}}{\partial z}=0 \\
\frac{\partial\left(u_{x}^{2}\right)}{\partial x}+\frac{\partial\left(u_{y} u_{x}\right)}{\partial y}+\frac{\partial\left(u_{z} u_{x}\right)}{\partial z}+\frac{1}{\rho} \frac{\partial p}{\partial x}=0 \\
\frac{\partial\left(u_{x} u_{y}\right)}{\partial x}+\frac{\partial\left(u_{y}^{2}\right)}{\partial y}+\frac{\partial\left(u_{z} u_{y}\right)}{\partial z}+\frac{1}{\rho} \frac{\partial p}{\partial y}=0
\end{gathered}
$$




$$
\frac{\partial\left(u_{x} u_{z}\right)}{\partial x}+\frac{\partial\left(u_{y} u_{z}\right)}{\partial y}+\frac{\partial\left(u_{z}^{2}\right)}{\partial z}+\frac{1}{\rho} \frac{\partial p}{\partial z}=0 .
$$

Системы (1.3) - (1.6) и (1.7) - (1.10) замкнуты относительно неизвестных функций - составляющих вектора скорости $u_{x}=u_{x}(x, y, z), u_{y}=u_{y}(x, y, z), u_{z}=u_{z}(x, y, z)$ и давления $p=p(x, y, z)$.

Займемся построением точных решений системы Навье-Стокса (1.3) - (1.6), не удовлетворяющих уравнениям Эйлера $(1.7)-(1.10)$. Будем искать такое решение в виде

$$
\begin{gathered}
u_{x}=0, \quad u_{y}=0, \quad u_{z}=A x^{2}+B y^{2}+\varphi(x, y), \\
p=p(z) .
\end{gathered}
$$

Здесь $A$ и $B$ - некоторые вещественные константы, подчиняющиеся условию $A+B \neq 0$. Функция $\varphi(x, y)$ является гармонической в некоторой области $V \subset \mathbb{R}_{x, y}^{2}$. Подстановка $(1.11)$ - (1.12) в (1.3) - (1.5) приводит к истинным равенствам. Из (1.6) находим

$$
\frac{1}{\rho} \frac{d p}{d z}=2 \nu(A+B) .
$$

Интегрирование (1.13) по переменной $z$ дает

$$
p=2 \nu \rho(A+B) z+p_{0},
$$

где $p_{0}=$ const. Поскольку $A+B \neq 0$, вязкий член в правой части (1.6) не обращается в ноль. Следовательно, набор функций (1.12), (1.14) не образует решение системы Эйлера (1.7) - (1.10).

Еще один класс точных решений будем искать в виде

$$
\begin{gathered}
u_{x}=A_{1} z^{2}+B_{1} z+C_{1}, \quad u_{y}=A_{2} z^{2}+B_{2} z+C_{2}, \quad u_{z}=0, \\
p=p(x, y) .
\end{gathered}
$$

Постоянные $A_{i}, B_{i}$ и $C_{i}, i=1,2$, таковы, что $A_{1}^{2}+A_{2}^{2}>0$. Уравнения (1.3) и (1.6) удовлетворяются тождественно. Из (1.4) и (1.5) находим

$$
\begin{aligned}
& \frac{1}{\rho} \frac{\partial p}{\partial x}=2 \nu A_{1}, \\
& \frac{1}{\rho} \frac{\partial p}{\partial y}=2 \nu A_{2} .
\end{aligned}
$$

В силу ограничения $A_{1}^{2}+A_{2}^{2}>0$ правая часть в одном из уравнений (1.4) или (1.5) не обращается в ноль. Из (1.17) и (1.18) находим

$$
p=2 \nu \rho\left(A_{1} x+A_{2} y\right)+p_{0},
$$

где $p_{0}=$ const. Еще одно точное решение системы Навье-Стокса, не удовлетворяющее уравнениям Эйлера, построено. 
Другое решение стационарной системы Навье-Стокса, описывающее вязкое течение в цилиндрических координатах, приведено в [6]. Оно может быть записано в декартовых координатах:

$$
\begin{gathered}
u_{x}=-\Omega y-\frac{\Gamma}{2 \pi} \frac{y}{x^{2}+y^{2}}, \quad u_{y}=\Omega x+\frac{\Gamma}{2 \pi} \frac{x}{x^{2}+y^{2}}, \\
u_{z}=\frac{A}{4 \nu \rho}\left(x^{2}+y^{2}\right)+B \ln \sqrt{x^{2}+y^{2}}+C, \\
p=\frac{\rho \Omega^{2}}{2}\left(x^{2}+y^{2}\right)+\frac{\rho \Omega \Gamma}{\pi} \ln \sqrt{x^{2}+y^{2}}-\frac{\rho \Gamma^{2}}{8 \pi^{2}\left(x^{2}+y^{2}\right)}+A z+p_{0} .
\end{gathered}
$$

Здесь $\Omega, \Gamma, A, B, C$ и $p_{0}-$ постоянные величины. Одна из констант $\Omega$ или Г отлична от нуля. Постоянная $A$ не равна нулю.

\section{2. Точные решения стационарной квазигидродинамической системы}

Квазигидродинамическая система, описывающая установившиеся течения слабосжимаемой вязкой жидкости, имеет вид

$$
\operatorname{div} \vec{u}=\operatorname{div} \vec{w}
$$

$$
\operatorname{div}(\vec{u} \otimes \vec{u})+\frac{1}{\rho} \nabla p=2 \nu \operatorname{div} \widehat{\sigma}+\operatorname{div}[(\vec{w} \otimes \vec{u})+(\vec{u} \otimes \vec{w})] .
$$

Обозначения стандартные. Влияние внешних сил не учитывается. Тензор скоростей деформаций определяется с помощью выражения

$$
\widehat{\sigma}=\widehat{\sigma}(\vec{u})=\frac{1}{2}\left[(\nabla \otimes \vec{u})+(\nabla \otimes \vec{u})^{T}\right] .
$$

Вектор $\vec{w}$ вычисляется по формуле

$$
\vec{w}=\tau\left((\vec{u} \cdot \nabla) \vec{u}+\frac{1}{\rho} \nabla p\right)
$$

где

$$
\tau=\frac{\nu}{c_{s}^{2}}
$$

- характерное время релаксации, $c_{s}$ - скорость звука в жидкости. Эквивалентная запись (2.1) - (2.2) такова:

$$
\begin{gathered}
\operatorname{div} \vec{u}=\operatorname{div} \vec{w}, \\
\operatorname{div}(\vec{u} \otimes \vec{u})+\frac{1}{\rho} \nabla p-\nu \Delta \vec{u}= \\
=\nu \nabla(\operatorname{div} \vec{u})+\vec{u} \operatorname{div} \vec{w}+\vec{w} \operatorname{div} \vec{u}+(\vec{w} \cdot \nabla) \vec{u}+(\vec{u} \cdot \nabla) \vec{w} .
\end{gathered}
$$

В декартовых координатах система (2.1) - (2.2) имеет вид

$$
\frac{\partial u_{x}}{\partial x}+\frac{\partial u_{y}}{\partial y}+\frac{\partial u_{z}}{\partial z}=\frac{\partial w_{x}}{\partial x}+\frac{\partial w_{y}}{\partial y}+\frac{\partial w_{z}}{\partial z},
$$




$$
\begin{gathered}
\frac{\partial\left(u_{x}^{2}\right)}{\partial x}+\frac{\partial\left(u_{y} u_{x}\right)}{\partial y}+\frac{\partial\left(u_{z} u_{x}\right)}{\partial z}+\frac{1}{\rho} \frac{\partial p}{\partial x}= \\
=\nu\left(2 \frac{\partial^{2} u_{x}}{\partial x^{2}}+\frac{\partial^{2} u_{x}}{\partial y^{2}}+\frac{\partial^{2} u_{x}}{\partial z^{2}}+\frac{\partial^{2} u_{y}}{\partial y \partial x}+\frac{\partial^{2} u_{z}}{\partial z \partial x}\right)+ \\
+2 \frac{\partial\left(w_{x} u_{x}\right)}{\partial x}+\frac{\partial\left(w_{y} u_{x}\right)}{\partial y}+\frac{\partial\left(w_{z} u_{x}\right)}{\partial z}+\frac{\partial\left(u_{y} w_{x}\right)}{\partial y}+\frac{\partial\left(u_{z} w_{x}\right)}{\partial z}, \\
\frac{\partial\left(u_{x} u_{y}\right)}{\partial x}+\frac{\partial\left(u_{y}^{2}\right)}{\partial y}+\frac{\partial\left(u_{z} u_{y}\right)}{\partial z}+\frac{1}{\rho} \frac{\partial p}{\partial y}= \\
=\nu\left(\frac{\partial^{2} u_{y}}{\partial x^{2}}+2 \frac{\partial^{2} u_{y}}{\partial y^{2}}+\frac{\partial^{2} u_{y}}{\partial z^{2}}+\frac{\partial^{2} u_{x}}{\partial x \partial y}+\frac{\partial^{2} u_{z}}{\partial z \partial y}\right)+ \\
\frac{\partial\left(w_{x} u_{y}\right)}{\partial x}+2 \frac{\partial\left(w_{y} u_{y}\right)}{\partial y}+\frac{\partial\left(w_{z} u_{y}\right)}{\partial z}+\frac{\partial\left(u_{x} w_{y}\right)}{\partial x}+\frac{\partial\left(u_{z} w_{y}\right)}{\partial z} \\
\frac{\partial\left(u_{x} u_{z}\right)}{\partial x}+\frac{\partial\left(u_{y} u_{z}\right)}{\partial y}+\frac{\partial\left(u_{z}^{2}\right)}{\partial z}+\frac{1}{\rho} \frac{\partial p}{\partial z}= \\
\nu\left(\frac{\partial^{2} u_{z}}{\partial x^{2}}+\frac{\partial^{2} u_{z}}{\partial y^{2}}+2 \frac{\partial^{2} u_{z}}{\partial z^{2}}+\frac{\partial^{2} u_{x}}{\partial x \partial z}+\frac{\partial^{2} u_{y}}{\partial y \partial z}\right)+ \\
+\frac{\partial\left(w_{x} u_{z}\right)}{\partial x}+\frac{\partial\left(w_{y} u_{z}\right)}{\partial y}+2 \frac{\partial\left(w_{z} u_{z}\right)}{\partial z}+\frac{\partial\left(u_{x} w_{z}\right)}{\partial x}+\frac{\partial\left(u_{y} w_{z}\right)}{\partial y} .
\end{gathered}
$$

Здесь

$$
\begin{aligned}
& w_{x}=\tau\left(u_{x} \frac{\partial u_{x}}{\partial x}+u_{y} \frac{\partial u_{x}}{\partial y}+u_{z} \frac{\partial u_{x}}{\partial z}+\frac{1}{\rho} \frac{\partial p}{\partial x}\right), \\
& w_{y}=\tau\left(u_{x} \frac{\partial u_{y}}{\partial x}+u_{y} \frac{\partial u_{y}}{\partial y}+u_{z} \frac{\partial u_{y}}{\partial z}+\frac{1}{\rho} \frac{\partial p}{\partial y}\right), \\
& w_{z}=\tau\left(u_{x} \frac{\partial u_{z}}{\partial x}+u_{y} \frac{\partial u_{z}}{\partial y}+u_{z} \frac{\partial u_{z}}{\partial z}+\frac{1}{\rho} \frac{\partial p}{\partial z}\right) .
\end{aligned}
$$

Покажем, что построенные в предыдущем пункте наборы функций $\left(u_{x}, u_{y}, u_{z}\right.$, $p$ ) также удовлетворяют системе (2.7) - (2.10).

В первом случае $\operatorname{div} \vec{u}=0$. Вектор $\vec{w}$ является постоянным:

$$
\vec{w}=\left(\begin{array}{c}
w_{x} \\
w_{y} \\
w_{z}
\end{array}\right)=\tau\left(\begin{array}{l}
u_{x} \frac{\partial u_{x}}{\partial x}+u_{y} \frac{\partial u_{x}}{\partial y}+u_{z} \frac{\partial u_{x}}{\partial z}+\frac{1}{\rho} \frac{\partial p}{\partial x} \\
u_{x} \frac{\partial u_{y}}{\partial x}+u_{y} \frac{\partial u_{y}}{\partial y}+u_{z} \frac{\partial u_{y}}{\partial z}+\frac{1}{\rho} \frac{\partial p}{\partial y} \\
u_{x} \frac{\partial u_{z}}{\partial x}+u_{y} \frac{\partial u_{z}}{\partial y}+u_{z} \frac{\partial u_{z}}{\partial z}+\frac{1}{\rho} \frac{\partial p}{\partial z}
\end{array}\right)=\left(\begin{array}{c}
0 \\
0 \\
2 \nu \tau(A+B)
\end{array}\right) .
$$


Поскольку

$$
(\vec{w} \cdot \nabla) \vec{u}=2 \nu \tau(A+B) \frac{\partial}{\partial z}\left(\begin{array}{c}
0 \\
0 \\
A x^{2}+B y^{2}+\varphi(x, y)
\end{array}\right)=0
$$

правые части (2.5) - (2.6) обращаются в ноль.

Во втором случае $\operatorname{div} \vec{u}=0$. Вектор $\vec{w}$ также является постоянным:

$$
\vec{w}=\left(\begin{array}{c}
w_{x} \\
w_{y} \\
w_{z}
\end{array}\right)=\tau\left(\begin{array}{c}
u_{x} \frac{\partial u_{x}}{\partial x}+u_{y} \frac{\partial u_{x}}{\partial y}+u_{z} \frac{\partial u_{x}}{\partial z}+\frac{1}{\rho} \frac{\partial p}{\partial x} \\
u_{x} \frac{\partial u_{y}}{\partial x}+u_{y} \frac{\partial u_{y}}{\partial y}+u_{z} \frac{\partial u_{y}}{\partial z}+\frac{1}{\rho} \frac{\partial p}{\partial y} \\
u_{x} \frac{\partial u_{z}}{\partial x}+u_{y} \frac{\partial u_{z}}{\partial y}+u_{z} \frac{\partial u_{z}}{\partial z}+\frac{1}{\rho} \frac{\partial p}{\partial z}
\end{array}\right)=\left(\begin{array}{c}
2 \nu \tau A_{1} \\
2 \nu \tau A_{2} \\
0
\end{array}\right) .
$$

Кроме того,

$$
(\vec{w} \cdot \nabla) \vec{u}=2 \nu \tau\left(A_{1} \frac{\partial}{\partial x}+A_{2} \frac{\partial}{\partial y}\right)\left(\begin{array}{c}
A_{1} z^{2}+B_{1} z+C_{1} \\
A_{2} z^{2}+B_{2} z+C_{2} \\
0
\end{array}\right)=0 .
$$

Поэтому правые части $(2.5)-(2.6)$ равны нулю.

В третьем случае $\operatorname{div} \vec{u}=0$. Постоянный вектор $\vec{w}$ вычисляется по формуле

$$
\vec{w}=\left(\begin{array}{c}
w_{x} \\
w_{y} \\
w_{z}
\end{array}\right)=\tau\left(\begin{array}{l}
u_{x} \frac{\partial u_{x}}{\partial x}+u_{y} \frac{\partial u_{x}}{\partial y}+u_{z} \frac{\partial u_{x}}{\partial z}+\frac{1}{\rho} \frac{\partial p}{\partial x} \\
u_{x} \frac{\partial u_{y}}{\partial x}+u_{y} \frac{\partial u_{y}}{\partial y}+u_{z} \frac{\partial u_{y}}{\partial z}+\frac{1}{\rho} \frac{\partial p}{\partial y} \\
u_{x} \frac{\partial u_{z}}{\partial x}+u_{y} \frac{\partial u_{z}}{\partial y}+u_{z} \frac{\partial u_{z}}{\partial z}+\frac{1}{\rho} \frac{\partial p}{\partial z}
\end{array}\right)=\left(\begin{array}{c}
0 \\
0 \\
\frac{\tau A}{\rho}
\end{array}\right) .
$$

Имеем

$$
(\vec{w} \cdot \nabla) \vec{u}=\frac{\tau A}{\rho} \frac{\partial}{\partial z}\left(\begin{array}{c}
-\Omega y-\frac{\Gamma}{2 \pi} \frac{y}{x^{2}+y^{2}} \\
\Omega x+\frac{\Gamma}{2 \pi} \frac{x}{x^{2}+y^{2}} \\
\frac{A}{4 \nu \rho}\left(x^{2}+y^{2}\right)+B \ln \sqrt{x^{2}+y^{2}}+C
\end{array}\right)=0 .
$$

Правые части $(2.5)-(2.6)$ обращаются в ноль.

\section{3. Примеры течений}

Приведем примеры течений, отвечающих построенным решениям систем Навье-Стокса и КГД.

Пример 1. Рассмотрим движение жидкости между двумя коаксиальными цилиндрами радиусов $R_{1}$ и $R_{2}$, где $0<R_{1}<R_{2}$. Ось $о z$ правой декартовой системы 
координат направим по оси цилиндров. Внутренний цилиндр неподвижен, внешний движется вдоль оси $о z$ с постоянной скоростью $U$. Значения давления в плоскостях $z=z_{1}=0$ и $z=z_{2}=L>0$ постоянно и равно соответственно $p_{1}$ и $p_{2}$. Числа $p_{1}$ и $p_{2}$ положительны, разность $\Delta p=p_{1}-p_{2}>0$. В промежутке между точками $z_{1}$ и $z_{2}$ давление $p=p(z)$ меняется по линейному закону

$$
p=p_{1}-\frac{\Delta p}{L} z .
$$

$\mathrm{B}(1.11)$ положим $A=B, \varphi(x, y)=c_{1} \ln \sqrt{x^{2}+y^{2}}+c_{2}$, где $c_{1}$ и $c_{2}$ - постоянные величины. Заметим, что функция $\varphi(x, y)$ является гармонической при $(x, y) \neq(0,0)$. Из (1.14) и (3.1) находим

$$
A=-\frac{\Delta p}{4 \nu \rho L}
$$

Константы $c_{1}$ и $c_{2}$ определим из граничных условий

$$
\left.u_{z}\right|_{x^{2}+y^{2}=R_{1}^{2}}=0,\left.\quad u_{z}\right|_{x^{2}+y^{2}=R_{2}^{2}}=U
$$

Распределение скорости принимает вид

$$
\begin{gathered}
u_{x}=0, \quad u_{y}=0 \\
u_{z}=\frac{\Delta p}{4 \nu \rho L}\left(R_{1}^{2}-x^{2}-y^{2}\right)+\frac{U+\frac{\Delta p}{4 \nu \rho L}\left(R_{2}^{2}-R_{1}^{2}\right)}{\ln \frac{R_{2}}{R_{1}}} \ln \left(\frac{\sqrt{x^{2}+y^{2}}}{R_{1}}\right) .
\end{gathered}
$$

В области $V_{1}=\left\{(x, y, z): R_{1}<\sqrt{x^{2}+y^{2}}<R_{2}, 0<z<L\right\}$ формулы (3.2), (3.1) описывают течение Куэтта-Пуазейля между коаксиальными соосными цилиндрами.

Пример 2. Пусть жидкость движется между двумя параллельными пластинами, расстояние между которыми равно $H>0$. Плоскость хоу правой декартовой системы координат свяжем с неподвижной нижней пластиной. Ось oz направим вертикально вверх. Вторая пластина с координатой $z=H$ движется с постоянной скоростью $V$ в направлении оси оу. Значения давления в плоскостях $x=x_{1}=0$ и $x=x_{2}=L>0$ постоянны и равны $p_{1}$ и $p_{2}$ соответственно, где $p_{1}$ и $p_{2}-$ положительные числа. На промежутке $\left[x_{1}, x_{2}\right]$ величина $p$ меняется по линейному закону:

$$
p=p_{1}-\frac{\Delta p}{L} x
$$

причем $\Delta p=p_{1}-p_{2}>0$. Из (1.17) и (1.18) находим

$$
A_{1}=-\frac{\Delta p}{2 \nu \rho L}, \quad A_{2}=0 .
$$

Распределение скорости (1.15) в области

$$
V_{2}=\{(x, y, z): 0<x<L,-\infty<y<+\infty, 0<z<H\},
$$


отвечающее такой физической постановке, выглядит следующим образом:

$$
u_{x}=\frac{\Delta p}{2 \nu \rho L} z(H-z), \quad u_{y}=\frac{V}{H} z, \quad u_{z}=0 .
$$

Движение жидкости, описываемое формулами (3.4) и (3.3), представляет собой композицию течений Пуазейля вдоль оси ox и Куэтта вдоль оси оy.

Пример 3. Рассмотрим цилиндрическую трубу радиуса $R>0$, вращающуюся вокруг своей оси с постоянной угловой скоростью $\Omega$. Направим ось $о z$ правой декартовой системы координат по оси трубы. Непосредственной проверкой можно убедиться в том, что функции

$$
\begin{gathered}
u_{x}=-\Omega y, \quad u_{y}=\Omega x, \quad u_{z}=\frac{\Delta p}{4 \nu \rho L}\left(R^{2}-x^{2}-y^{2}\right), \\
p=\frac{\rho \Omega^{2}}{2}\left(x^{2}+y^{2}\right)+p_{1}-\frac{\Delta p}{L} z,
\end{gathered}
$$

где $p_{1}>0, p_{2}>0$ и $\Delta p=p_{1}-p_{2}>0$, в области

$$
V_{3}=\left\{(x, y, z): 0 \leqslant \sqrt{x^{2}+y^{2}}<R, 0<z<L\right\}
$$

удовлетворяют системе Навье-Стокса (1.3) - (1.6) и квазигидродинамической системе (2.7) - (2.10). Они отвечают течению Пуазейля во вращающейся трубе. Однако набор функций $(3.5),(3.6)$ из класса $(1.20),(1.21)$ не является точным решением уравнений Эйлера $(1.7)-(1.10)$.

\section{Заключение}

Особый интерес представляют точные решения стационарных квазигидродинамических уравнений, не удовлетворяющие системам Эйлера и Навье-Стокса. Такие решения существуют (см. [4], с. 106 - 107). Они зависят от положительного параметра $\tau$ и их построение в большинстве задач сопряжено со значительными трудностями. В каких случаях решение системы Навье-Стокса может быть получено из соответствующего решения системы КГД предельным переходом при $\tau \rightarrow+0$ ? Ответа на этот сложный вопрос пока нет.

\section{Список литературы}

[1] Ландау Л.Д., Лифшиц Е.М. Гидродинамика. М.: Наука, 1986. 736 с.

[2] Лойцянский Л.Г. Механика жидкости и газа. М.: Наука, 1987. 840 с.

[3] Шеретов Ю.В. О единственности решений одной диссипативной системы гидродинамического типа // Математическое моделирование. 1994. Т. 6, № 10. C. $35-45$.

[4] Шеретов Ю.В. Динамика сплошных сред при пространственно-временном осреднении. М., Ижевск: НИЦ «Регулярная и хаотическая динамика», 2009. 400 c. 
[5] Шеретов Ю.В. Регуляризованные уравнения гидродинамики. Тверь: Тверской гос. ун-т, 2016. 222 с.

[6] Шеретов Ю.В. О точных решениях стационарных квазигидродинамических уравнений в цилиндрических координатах // Вестник ТвГУ. Серия: Прикладная математика. 2017. № 1. С. 85-94.

[7] Жериков А.В. Применение квазигидродинамических уравнений: математическое моделирование течений вязкой несжимаемой жидкости. Saarbrücken: Lambert Academic Publishing, 2010. 124 c.

[8] Елизарова Т.Г., Милюкова О.Ю. Численное моделирование течений вязкой несжимаемой жидкости в кубической каверне // Журнал вычислительной математики и математической физики. 2003. Т. 43, № 3. С. 453-466.

\section{Библиографическая ссылка}

Шеретов Ю.В. Об общих точных решениях стационарной системы НавьеСтокса и квазигидродинамической системы, не удовлетворяющих уравнениям Эйлера // Вестник ТвГУ. Серия: Прикладная математика. 2017. № 2. С. 5-15.

\section{Сведения об авторах}

\section{1. Шеретов Юрий Владимирович}

заведующий кафедрой математического анализа Тверского государственного университета.

Россия, 170100, г. Тверь, ул. Желябова, д. З3, ТвГУ.

E-mail: Sheretov.YV@tversu.ru. 


\title{
ON THE COMMON EXACT SOLUTIONS OF STATIONARY NAVIER-STOKES AND QUASI-HYDRODYNAMIC SYSTEMS, NOT SATISFYING TO EULER EQUATIONS
}

\author{
Sheretov Yurii Vladimirovich
}

Head of Mathematical Analysis department, Tver State University. Russia, 170100, Tver, 33 Zhelyabova str., TSU. E-mail: Sheretov.YV@tversu.ru

Received 21.03.2017, revised 27.04.2017.

Three families of exact solutions, which are common for the stationary Navier-Stokes system and corresponding quasi-hydrodynamic system, are constructed. These solutions do not satisfy to Euler equations. The concrete examples of solutions that describe the flows of a viscous fluid are presented. Their physical interpretation is given.

Keywords: Navier-Stokes and Euler systems, quasi-hydrodynamic equations, exact solutions.

\section{Bibliographic citation}

Sheretov Yu.V. On the common exact solutions of stationary Navier-Stokes and quasi-hydrodynamic systems, not satisfying to Euler equations. Vestnik TvGU. Seriya: Prikladnaya Matematika [Herald of Tver State University. Series: Applied Mathematics], 2017, no. 2, pp. 5-15. (in Russian)

\section{References}

[1] Landau L.D., Lifshits E.M. Gidrodinamika [Hydrodynamics]. "Nauka" Publ., Moscow, 1986. 736 p. (in Russian)

[2] Loytsyansky L.G. Mekhanika Zhidkosti i Gaza [Fluid and Gas Mechanics]. "Nauka" Publ., Moscow, 1987. 840 p. (in Russian)

[3] Sheretov Yu.V. On uniqueness of the solutions for one dissipative system of hydrodynamic type. Matematicheskoe Modelirovanie [Mathematical Modeling], 1994, vol. 6(10), pp. 35-45. (in Russian)

[4] Sheretov Yu.V. Dinamika Sploshnykh Sred pri Prostranstvenno-Vremennom Osrednenii [Continuum Dynamics under Spatiotemporal Averaging]. "Regular and Chaotic Dynamics" Publ., Moscow, Izhevsk, 2009. 400 p. (in Russian)

[5] Sheretov Yu.V. Regulyarizovannye Uravneniya Gidrodinamiki [Regularized Hydrodynamic Equations]. Tver State University, Tver, 2016. 222 p. (in Russian) 
[6] Sheretov Yu.V. On the exact solutions of stationary quasi-hydrodynamic equations in cylindrical coordinates. Vestnik TvGU. Seriya: Prikladnaya matematika [Herald of Tver State University. Series: Applied Mathematics], 2017, no. 1, pp. 85-94. (in Russian)

[7] Zherikov A.V. Primenenie Kvazigidrodinamicheskikh Uravnenii: Matematicheskoe Modelirovanie Techeniy Vyazkoi Neszhimaemoi Zhidkosti [Application of QuasiHydrodynamic Equations: Mathematical Modeling of Viscous Incompressible Fluid]. Lambert Academic Publishing, Saarbrücken, 2010. 124 p. (in Russian)

[8] Elizarova T.G., Milyukova O.Yu. Numerical simulation of viscous incompressible flow in a cubic cavity. Computational Mathematics and Mathematical Physics, 2003, vol. 43(3), pp. 433-445. 